Review

\title{
Triage systems: a review of the literature with reference to Saudi Arabia
}

\author{
N.A. Qureshi ${ }^{7}$
}

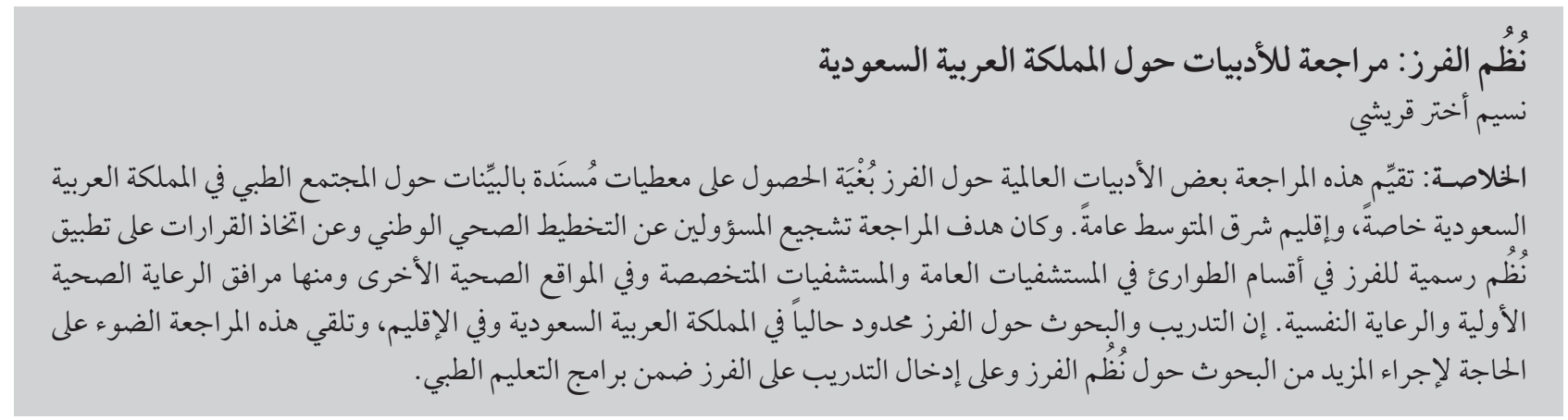

ABSTRACT This review evaluates some of the international literature on triage in order to provide evidencebased data for the medical community in Saudi Arabia specifically and the Eastern Mediterranean Region in general. The aim is to encourage national health planners and decision-makers to apply formal triage systems in the emergency departments of general and specialist hospitals and other relevant health settings, including primary and psychiatric care. Research and training on triage is extremely limited in Saudi Arabia and the Region and this review highlights the need for more research on triage systems and for the inclusion of training on triage in medical education programmes.

\section{Systèmes de triage : revue de la littérature et référence à l'Arabie saoudite}

RÉSUMÉ Cette revue évalue une partie de la littérature internationale concernant le triage afin de fournir des données factuelles à la communauté médicale saoudienne en particulier et de la Région de la Méditerranée orientale en général. Le but est d'encourager les planificateurs sanitaires et les décideurs nationaux à mettre en place des systèmes de triage formels dans les services des urgences des hôpitaux généraux et spécialisés, ainsi que dans d'autres structures sanitaires qui en ont besoin, notamment les unités de soins de santé primaires et de soins psychiatriques. La recherche et la formation en matière de triage sont extrêmement limitées en Arabie saoudite et dans la région, et cette revue met en avant la nécessité d'une intensification de la recherche sur les systèmes de triage et de l'intégration d'une formation sur le triage dans les programmes d'enseignement médical. 


\section{Introduction}

The hospital emergency department (ED) is one of the most important components of the health delivery system. EDs worldwide are reportedly serving increasing numbers of patients who have a range of problems of variable urgency, from life-threatening to mild. Studies have found that around 50\% of ED visits are for nonurgent reasons, leading to unnecessary costs and multiple adverse consequences $[1-5]$. All patients arriving for emergency care need to be assessed and classified to prioritize those who have the most urgent medical problems and are in need of immediate care. This classification process is termed triage and it is usually performed by an ED nurse. The aim of triage is to distribute the workload of the ED for better utilization of resources.

In Saudi Arabia, as elsewhere, improving the utilization of EDs is the subject of research and debate $[6,7]$. This review evaluates some of the international literature on triage in order to provide evidence-based data for the medical community in Saudi Arabia specifically and the Eastern Mediterranean region (EMR) in general. The aim is to encourage national health planners and decision-makers to apply formal triage systems in the EDs of general and specialist hospitals and other relevant health settings, including primary and psychiatric care. Research and training on triage is extremely limited in Saudi Arabia and the Region and this review hopes to stimulate more research on triage systems and to make the case for the inclusion of training on triage in medical education programmes.

\section{Definition of triage}

Triage is the process of categorizing ED patients according to their need for medical care, irrespective of their order of arrival or other factors including sex, age, socioeconomic status, insurance status, residential status, nationality, race, ethnicity or religion. Triage involves an assessment to prioritize ED patients in need of immediate care, in accordance with clinical severity and time urgency, compared with patients with nonurgent illnesses who can wait longer to be seen or who need referral to a more appropriate health care setting [8].

Different methods of triage are practised; in-person triage (direct) has been shown to be preferable to telephone triage (indirect) [9], because the latter involves imaginary picture building or "visualization work" [10]. One study, however, has shown that telephone triage by nurses did not delay treatment interventions when compared to triage by doctors in a paediatric setting [3]. Telephone triage is commonly used in psychiatric settings [11].

The process of triage decisionmaking is influenced by 3 interrelated factors: the characteristics of the patient, the triage decision-maker and the health care setting [12]. Triage is important for redistributing and reducing waiting times and admission rates, increasing the efficiency and effectiveness of the $\mathrm{ED}$, enhancing patient and family satisfaction, improving the quality of health care, managing funding and assessing the effectiveness of ED activities [12].

While the importance of triage in the ED has been recognized for some time in developed countries, less developed countries, including those of the EMR, are not utilizing the full potential of this health developmental trend. The EDs of psychiatric hospitals especially have much progress to make to realize the benefits of triage.

\section{Triage and health professionals}

Triage is usually performed in the ED by nursing staff who allocate a triage designation and initiate emergency care before the patient is examined by a doctor. Triage may be done by ED physicians as well. In prehospital disaster sites, ambulance personnel also need to use triage to prioritize multiple cases for immediate mass evacuation for emergency medical help in nearby hospitals.

Triage nurses usually have advanced training in decision-making. They have been shown to have the necessary skills to make appropriate triage decisions and provide a highly effective service to ED patients in health care settings [13-16]. Many patients arriving at the ED have complex problems that need several investigations, procedures or consultations. Triage nurses can validly and reliably estimate the complexity of such cases, guide ED workflow and casemix system analysis [17].

In a study of decisions about appropriate care provider, priority rating and preliminary investigations for ambulatory patients the level of agreement between the triage nurses and physician observers was $81 \%$ and between the triage nurses and treating physicians was 94\% [18]. Triage nurses identified a greater number of patients (19\%) as having emergency problems (17\%), and fewer patients (45\%) as having problems of a non-urgent nature compared with physician observers (47\%). The study concluded that experienced emergency nurses in the role of triage were safe, efficient and cost-effective, with statistically significant levels of safety and accuracy of priority rating when compared to triage physicians and treating physicians.

The increasing use of triage and the increasing numbers of $\mathrm{ED}$ visits by patients raises the important issue of a parallel need to increase the number of triage nurses in EDs [19,20].

\section{Triage scales}

Different triage scales have been developed to help health professionals to classify ED patients consistently and to achieve acceptable health outcomes. 
Triage scales usually have 3 to 5 categories, with algorithms or protocols or sentinel diagnoses as the anchor points for making decisions, supported by triage guidelines and procedures. They are now generally supported by computerbased programs and web-sites which are faster and more effective aids to prioritization and decision-making.

The most commonly used scale is the Australian Triage Scale (ATS), which has 5 categories with their corresponding level of treatment acuity (Table 1). The ATS is derived from the National Triage Scale for Australasian Emergency Departments (NTS), the 2 scales differing in the description and allocation of the 5 categories. In a study in Belgium, the NTS was reported to have good predictive validity [21]. Yousif et al. reported that use of the ATS had a significant impact on the triage distribution of ED patients compared with the NTS, with $28 \%$ and $24 \%$ increases in patients with categories 2 and 3 respectively and $15 \%$ and $67 \%$ decreases in patients with categories 4 and 5, respectively. The ATS is therefore better suited to meet performance criteria and casemix assessment [22].

Another scale from Australia is the Toowoomba Adult Triage Trauma Tool (TATTT), which is a computerized algorithmic clinical-decision support tool designed for use on a handheld personal computer. It is well accepted by users and is seen as a viable alternative to current triage practice [23]. The TATT incorporates ATS categories but largely replaces the associated clinical indicators and provides an evidence-based valid and consistent method of triage assessment and categorization of trauma patients.

The other international triage scales in common use are the Manchester Triage System (MTS) with its new updated version, the Emergency Severity Index (ESI) used in the United States of America and the Canadian Emergency Department Triage and Acuity Scale (CTAS) and its web-based triage tool (eTRIAGE) [24-26].

Ambulance personnel use disaster triage based on the Homebush Triage Standard Taxonomy in prehospital settings, which includes battlefields, accident and trauma sites and places of massive fires [27,28].

Mental health triage scales have also been developed for triaging ED patients with mental disorders because the triage scales mentioned above have little capacity for triaging mental patients. Psychiatric nurses have been shown to use these scales effectively in the EDs of psychiatric hospitals $[29,30]$.

More detailed information on triage scales including digital-reference triage scales can be found in the literature [12,26-32].

\section{Triage outcomes}

There are 2 stages to the nurse triage process: first, the triage assessment which leads to allocation of a triage category and the subsequent processing of the patient; and secondly, the initiation of nursing interventions to facilitate emergency care with a possible reduction in the patient's discomfort. These triage decisions are linked with 3 types of outcome: "correct" or "expected" triage, "over-triage" and "under-triage". Correct triage by a nurse is associated with a positive health outcome because the patient is evaluated by a doctor within a suitable timeframe. Over-triage and under-triage indicate that triage nurse allocated a triage category of a higher or lower acuity than required respectively. Outcomes associated with over- or under-triage result in inappropriate allocation of ED resources, prolonged waiting times for patients, and development of dangerous complications or prolonging suffering. Notably, funding models or incentive programmes for triage are considered unethical $[33,34]$.

\section{Effect of triage on waiting times}

Nurse triage aims to redistribute the workload of the ED. The key issue is not increasing or reducing waiting times overall but the effectiveness with which triage nurses allocate shorter waiting times to the highest priority patients, thus redistributing patient waiting times according to need. Waiting times in the ED affect patients' satisfaction with care but may also have serious complications that adversely affect health outcomes $[33,34]$. Prolonged waiting times at triage sites are the most common reason for patients' and families' dissatisfaction with ED care [35]. Accordingly

\begin{tabular}{|c|c|c|}
\hline ATS category & Description of category & Treatment acuity \\
\hline 1 & Immediately life-threatening & Immediate \\
\hline 2 & Immediately life-threatening & Minutes $(<10 \mathrm{~min})$ \\
\hline 3 & $\begin{array}{l}\text { Important time-critical intervention; potentially life- } \\
\text { threatening; situational urgency }\end{array}$ & 0.5 hours \\
\hline 4 & $\begin{array}{l}\text { Potentially serious; situational urgency; significant } \\
\text { complexity or severity }\end{array}$ & 1 hour \\
\hline 5 & Less urgent; clinic-administrative problems & 2 hours \\
\hline
\end{tabular}


patients need to understand, perhaps via educational campaigns, which medical problems are appropriate to present to the ED and also be informed about the waiting times that correspond to the category of urgency they are allocated.

\section{Factors influencing triage decisions}

Triage decisions are governed by 6 steps: step 1, visual cues, step 2, chief complaints, step 3, focused assessment, step 4, hypothesis construction, step 5 , acuity determination and step 6 , reassessment of the acuity [36].

The assessment of patients in the ED by triage nurses has been the focus of a study that used in-person triage and telephone triage for allocating patients to categories [36]. The results of this 2-phase research indicated that with regard to triage decisions, agreement among triage-certified nurses was poor ( $43 \%$ to $49 \%$ ) and a knowledge of patients' vital data and the use of triage protocols did not further improve the level of agreement ( $44 \%$ to $46 \%$ ).

There is converging evidence that the level of activity in the ED does not impact greatly on triage decisions [37]. Triage decisions have been shown to be affected principally by patients' clinical characteristics and the number and combination of clinical features and to a lesser extent by other factors such as the skill mix of the nursing and medical staff, staff intuition and triage guidelines $[12,37,38]$ (Table 2). Triage protocols for specific medical problems such as musculoskeletal problems have also been developed that impact on triage decision-making [39]. However, the triage decision-making process is also affected by factors such as interruptions to the triage process, time constraints and high workload, nurse skills and lack of formal staff training in triage, personal capacity (internal factors) and types of examinations and tests used $[25,38,40]$.

\begin{tabular}{lcl}
\hline $\begin{array}{l}\text { Table } 2 \text { Factors influencing triage decisions according to emergency department } \\
\text { (ED) staff }\end{array}$ & $\begin{array}{c}\text { Influence on triage } \\
\text { decisions (\% of } \\
\text { participants) }\end{array}$ & Remarks \\
\hline Studied factor & 98 & Yes \\
Clinical condition of the patient & 49 & Yes \\
Significance of patient history & 28 & Yes \\
Potential for treatment & 15 & Yes \\
Mechanism of injury & 2 & Yes \\
Potential for self-discharge & 2 & Yes \\
Operational factors in ED & 3 & Equivocal \\
ED activity levels & 2 & Yes \\
Medical skill mix & 1 & Yes \\
Nursing skill mix & & No effect \\
Other factors & 0 & No effect \\
Type of hospital & 0 & No effect \\
Triage nurse experience & 0 & \\
ED activity &
\end{tabular}

aTeaching, non-teaching, rural, private.

Sources: Richardson, 1998 [38]; Whitby et al., 1997 [39].

\section{Psychometrics of triage scales}

Studies of the reliability and validity of triage scales have reported that they have poor $(<0.2)$ to substantial $(0.61-0.80)$ to very good $(0.81-1.00)$ inter- and intra-rater reliability, attributable both to the structure of the scales and factors influencing the decisions [12,31-33,36,41-44]. Triage scales with 5 categories have better reliability than those with only 3 [45].

Training on the use of triage scales has a variable role in increasing the reliable use of scales. In one study, training effected moderate to good agreement between study nurses and duty triage nurses, with a trend toward improvement in the reliability of use of triage scales with additional training [24].

The clinical characteristics of ED patients also contribute to the variable reliability of triage. Research shows that some clinical features are associated with lower agreement while others show higher agreement among triage nurses in the allocation of triage category (Table 3 ) and this issue has been explored extensively [12].
Triage of patients with aggressive behaviour and threats to others, which are major components of psychiatric emergencies, have a good level of agreement (kappa $>0.8$ ), whereas triage of patients with anxious behaviour has a low level of agreement (kappa $<0.4$ ) between triage nurses and allocation of triage category [12].

\section{Uses of triage scales}

Triage scales were developed initially to allow ED patients to be prioritized reliably and to enhance uniform delivery of health services across EDs of all hospitals, including psychiatric settings. Other uses of triage scales include assessment of the performance of the ED and the quality of health care and allocation of funds.

Prolonged waiting times indicate failure of both access and quality. Reduced patient waiting times can be used as an indicator of good quality care and better performance by the $\mathrm{ED}[12,46]$.

Nurse triage has been found to enhance patient satisfaction, which is another measure of health care quality $[46,47]$. Even telephone-based nurse 


\begin{tabular}{lcc}
\hline \multicolumn{3}{l}{ Table 3 Level of agreement between triage nurses and allocation of triage category } \\
\hline Clinical characteristic & \% agreement & Kappa statistic \\
Lower levels of agreement $(\boldsymbol{\kappa}<\boldsymbol{0 . 4})$ & 30.0 & 0.259 \\
Sweaty skin & 28.8 & 0.224 \\
Dry skin & 47.7 & 0.395 \\
Anxious behaviour & 64.2 & 0.282 \\
Normal speech & 33.3 & 0.277 \\
Irritability (paediatric) & 50.0 & 0.327 \\
Crying (paediatric) & 46.0 & 0.379 \\
Dull pain & 0.0 & -0.004 \\
Crushing pain & & \\
Good levels of agreement $(\boldsymbol{\kappa}>\boldsymbol{O . 8})$ & 100.0 & 1.0 \\
Aggressive behaviour & 100.0 & 1.0 \\
Threat to others & 100.0 & 0.875 \\
Lethargy (paediatric) & 87.8 & 0.822 \\
Abdominal pain & 85.3 & 0.921 \\
Pregnancy & 100.0 & 1.0 \\
Haematemesis & 100.0 & 1.0 \\
Malaena & 100.0 & 1.0 \\
Overdose, ingestion & 100.0 & 1.0 \\
Eye presentation & 100.0 & 1.0 \\
Seizures & & \\
\hline
\end{tabular}

Source: Whitby et al., 1997 [39].

triage services have high patient satisfaction that did not vary substantially by caller characteristics [48]. In another study using retrospective data, patients' satisfaction with telephone-based nurse triage services was very high in psychiatric nurse triage [11].

Triage scales can also be a tool for developing strategies such as bonus payments to departments or hospitals for improving the performance of ED. Somehospitals setstandards that require EDs to provide medical care to $100 \%$ of category 1 patients, $80 \%$ of category 2 patients, $75 \%$ of category 3 patients, and $70 \%$ of category 4 and 5 patients within their requisite timeframes. Studies have concluded that EDs should triage patients to lower triage categories to meet hospital waiting time targets $[12,49]$.

\section{Education for triage}

According to many studies, specialty education and continuing training in triage are the cornerstones of the triage system and contribute considerably to the correct triage decisions that are essential for good health outcomes [24,50,51]. Continuing training but not experience was found to influence triage decision-making $[13,14,40]$.

Prior to adopting a triage role, nurses should have both adequate specialist training and experience in the triage system [14]. Studies in the United States and Australia concluded that triage nurses have wide variability in their degree of experience, preparation and orientation for the triage role, and insufficient education and training. Hospitals also vary in their requirements for training and experience before triage duties are performed [50,52]. The researchers recommended that a triage nurse should fulfil several conditions: a minimum of 3 months' experience in the ED; educational preparation; Advanced Cardiac Life Support certification; certificate in emergency or critical care nursing; completion of training workbooks; inservice education sessions; unit-based orientation programmes; competency assessment programmes; and triage guidelines [50,52].

The EDs of general hospitals receive patients with mental problems and likewise the EDs of psychiatric hospitals receive patients with physical diseases and injuries and in these contexts triage nurses need appropriate training and skills both in physical and psychiatric emergencies. General hospital triage nurses identified the following learning areas in mental health: customer focus, workplace aggression and violence, psychiatric theory, mental health assessment and chemical dependence [53]. Researchers have recommended collaboration between an adult mental health programme and an adult emergency programme [54].

In view of the variability in triage training and experience, there is a worldwide need to develop uniformly tailored triage education curricula and triage guidelines, as well as continuing training and research in triage systems [24]. Triage guidelines coupled with triage education and training helps triage nurses to prioritize ER patients in all health care settings including psychiatric EDs $[11,55]$. No triage guidelines, however, are perfect in predicting which patients are true emergencies [56].

\section{Triage quality improvement}

The quality measures for triage systems that have been explored in several studies include the reliability of triage rating scales, waiting times, admission rates, accuracy of allocation of categories and rates of sentinel events/diagnoses (outcomes unrelated to the natural course of the patient's illness or underlying condition). Notably each quality indicator has some disadvantages, such as the use of sentinel diagnosis, which can be made only after extensive interviewing and evaluation, which adversely 
affects waiting times $[12,26,33,57,58]$. Inconsistency in application of various triage scales is another concern for quality improvement in triage systems [33]. Quality standards in ED can be maintained and enhanced by audits of the triage system. Notably, a study of the resource implications of nonurgent patients in the ED showed that $7.3 \%$ of all patients requiring admission came from the group identified as nonurgent by the CTAS triage system and hence strategies diverting them elsewhere might be unsafe and were unlikely to improve access for more urgent patients [59].

\section{Triage in Saudi Arabia}

The Ministry of Health $(\mathrm{MOH})$ in Saudi Arabia has compiled a manual for organizing nursing services in the country with nursing triage guidelines that are to be used across all $\mathrm{MOH}$ EDs [unpublished document, General Administration for Nursing ,2003].

This document specifies nursing functions and duties together with comprehensive policies and procedures. With regard to specialized emergency nursing care, the manual defines triage, highlights its objectives and policies, defines 3 levels of triage activity and specifies the required manpower, materials, equipment and procedures. Psychiatric triage is not specifically mentioned in the manual but these guidelines are applicable to psychiatric EDs. The 3 levels of triage activity defined are:

- Emergent (I): patients require immediate medical intervention for conditions such as compromised airway problems, cardiac arrest, severe shock, cervical spine injury, multisystem trauma, altered level of consciousness and eclampsia.

- Urgent (II): patients are stable and there is no immediate threat to the patient's life or limbs but associated conditions require medical intervention within a few hours.
- Management (III): patients present with chronic or minor injuries with no danger to life or limb together with no distress or discomfort and may wait hours before being seen by an ED doctor. Conditions include chronic low back pain, routine medication refills, dental problems and missed menses.

In the field triage of disasters such as major fires, road traffic accidents, wars and explosions, patients are to be categorized as follows; red, emergent; yellow, immediate; green, urgent; blue, fast track or psychological support needed; and black, dead or progressing rapidly towards death.

The manual outlines emergencyprocedures for stabilizing patients, checking and monitoring for vital signs plus neurological assessment with the Glasgow coma scale. After prioritization, an ED nurse is expected to do the following; history taking and head-to-toe assessment; diagnostic and laboratory tests; ECG monitoring; cleansing and dressing of wounds; looking for suspected fractures; documenting all procedures in nurses' notes; and finally expedite admission or surgery if required. In psychiatric $\mathrm{ED}$, many of these procedures are not required.

The document does not mention adaptation of any international triage scale to be used in Saudi health settings apart from the 3-level system outlined above. However, some tertiary $\mathrm{MOH}$ hospitals such as Riyadh Medical Complex and King Fahad Medical City already use the CTAS system. Likewise some non-MOH hospitals such as King Faisal Specialist Hospital and Research Center, National Guard Hospital and Armed Forces Hospital and private hospitals use CTAS in their EDs. Triage is yet to be used in psychiatric settings in Saudi Arabia. The effectiveness of triage systems in these hospitals has not been evaluated. To our knowledge there is no published study that has systematically explored triage in the EMR and surrounding countries, although studies in Turkey, Saudi Arabia and Bahrain have evaluated the use of ED services, waiting times in the triage area, costeffectiveness, workload reduction and resource utilization of intensive care units $[7,60,61]$.

\section{Discussion}

Triage, applied strictly using standard principles in EDs, psychiatric settings and health disaster situations, remains a complex issue. Primary and secondary decisions by triage nurses and physicians and related health outcomes are influenced by a variety of internal factors related to triage personnel and external factors related to the operational mechanisms of the ED. Triage nurses use a constellation of factors to make triage decisions and initiate emergency care, including personal capacity, experience, intuition, prehospital information and communication with colleagues. In Saudi Arabia, nurses mostly have no involvement in triage decision-making. Yet the time has now come for health policy-makers to reflect seriously on the phased application of a nurse triage system across EDs in all health settings.

Many studies have shown that prioritization of ER patients is influenced by the clinical characteristics of patients, vital signs, severity of the condition and emerging complications $[31,36,42]$. The issue of frequent ED visits by patients might be solved, and patients' satisfaction improved, by managing patient waiting times better and briefing patients at presentation and at discharge [62]. Auditing triage decisions and consequent health outcomes is a useful activity as it addresses an important quality indicator of health care in ED settings [58]. Studies should also explore patients' perceptions of the triage system [63]. Patients' contribution to the triage decision is another area for research: a study in Saudi Arabia investigated the ability of adult patients to predict the presence or absence of 
fever in an ED triage clinic [64]. Mobile emergency triage and telephone-based triage, if applied in ED, could reduce the workload in EDs in a cost-effective way [65]. Internet "chat" as a triage method has been under evaluation in the United Kingdom [66]. Many researchers have advocated that available triage scales need to be improved with regard to their inter- and intra-rater reliability $[12,36,42]$ and computer-supported and web-based triage might overcome the psychometric weaknesses of the available scales [23].

Triage is a highly important activity and solves many problems of emergency services, such as overcrowding by patients, and improves the quality of health outcomes cost-effectively. Rising health care costs, poor quality of care and patient dissatisfaction are evident in settings where EDs are not applying triage [46]. Therefore triage needs to be applied in all EDs in Saudi Arabia and other EMR countries where there are adequate staff and other required resources $[19,20]$. Training of nurses in triage and continuous training for capacity-building has been repeatedly emphasized in the international research $[24,50,51]$. Use of triage in mental health delivery systems would also enhance consumers' satisfaction and solve the problem of aggressive and violent patients $[43,67,68]$. Nurse triage in mental health is less well researched.

\section{Challenges}

There are many challenges to introducing and developing ED triage in Saudi Arabia and elsewhere in the EMR.

- Developing public trust in the system. The sociocultural traditions of Saudi Arabia and other EMR countries do not conflict with the triage model. However, public awareness programmes about triage, compatible with social and cultural values, are needed. These need to promote the advantages including its vital role in improving patient survival and other health-related outcomes, satisfaction of patients and families and the cost-effectiveness and quality of health care.

- Capacity-building and recruitment of nurses. Recruiting qualified nurses with special training in triage can solve the initial problem of applying triage across entire nations. Such nurses have a role not only in the triage process but also in training other nurses for continuing capacity-building in the system. Achieving uniformity with regard to education and training of triage nurses is a constant challenge for health authorities and academics in different health settings even in high-income countries.
- Infrastructural developments in the ED. Appling triage may require additional equipment and space in the ED. This may not be a major issue in high-resource countries such as Saudi Arabia. Nevertheless it is advisable that triage should be applied to EDs in phases over a stipulated period of time. This should be accompanied by audits of health outcomes in EDs with and without triage.

\section{Future directions}

- Future research focussed on this region should assess how triage nurses prioritize ED patients, designate categories to individual patients and what criteria and information they use in doing so.

- Continuing training on triage systems for nurses and physicians will improve staff capacity-building in Saudi Arabia and elsewhere in the EMR. There should be continuing evaluation of the effectiveness of triage training programmes on the perceptions, practice and knowledge of triage nurses.

- Public campaigns are needed to build up public trust in the triage system with the emphasis on how patients are prioritized based on the severity of the problem at hand rather than other factors.

\section{References}

1. Baker LC, Baker LS. Excess cost of emergency department visits for nonurgent care. Datawatch, 1994, 13(5):162-71.

2. Doobinin KA et al. Nonurgent pediatric emergency department visits: care-seeking behavior and parental knowledge of insurance. Pediatric emergency care, 2003, 19:10-4.

3. Lee TJ et al. Does telephone triage delay significant medical treatment? Advice nurse service vs on-call pediatricians. Archives of pediatrics \& adolescent medicine, 2003, 157:635-41.

4. Kalemoglu M et al. Non-urgent patients in an emergency medical service. Revista medica de Chile, 2004, 132:1085-9.

5. Carter AJE, Chochinow AH. A systematic review of the impact of nurse practitioners on cost, quality of care, satisfaction and wait times in the emergency department. Canadian journal of emergency medicine, 2007, 9:286-95.
6. Qureshi NA et al. A study of utilization pattern of psychiatric emergency services (PES) in Al-Qassim Region, Saudi Arabia. Saudi medical journal, 1997, 18:137-43.

7. Al-Arabi Y, Al-Shimemen A. Improving resource utilization in intensive care units, a challenge for Saudi hospitals. Saudi medical journal, 2003, 24:131-7.

8. Porter JE. Nurse triage. British medical journal, 1993, 306:208-9.

9. Nakagawa J et al. Interobserver agreement in emergency department triage. Annals of emergency medicine, 2003, 41:191-5.

10. Edwards B. Seeing is believing-picture building: a key component of telephone triage. Journal of clinical nursing, 1998. 7:51-7.

11. Morrison A, Hull A, Shepherd B. Triage in emergency psychiatry. Psychiatric bulletin, 2000, 24:261-4. 
12. Whitby S et al. Analysis of the process of triage: the use and outcome of the National Triage Scale. Liverpool, Liverpool Health Service, 1997.

13. Cioffi J. Triage decision making: educational strategies. Accident and emergency nursing, 1999, 7:106-11.

14. George $\mathrm{S}$ et al. Nurse triage in theory and in practice. Archives of emergency medicine, 1993, 10:220-8.

15. Chorzempa A, LaMotte A. The role of the triage nurse practitioner in general medical practice: an analysis of the role. Journal of advanced nursing, 1998, Clinical excellence for nurse practitioners, 1999, 3(3):189-90.

16. Chan JN, Chau J. Patient satisfaction with triage nursing care in Hong Kong. Journal of advanced nursing, 2005, 50:498-507.

17. Vance J, Sprivulis P. Triage nurses validly and reliably estimate emergency department patient complexity. Emergency medicine Australasia, 2005, 17:382-6.

18. Zwicke DL, Bobzien WF, Wagner EF. Triage nurse decisions: a prospective study. Journal of emergency nursing, 1982, 8:132-8.

19. Aiken LH, Havens DS, Sloane DM. Magnet nursing services recognition programme. Nursing standard, 2000, 14:41-7.

20. O'Brien-Pallas L, Irvin D, Peereboom E. Measuring nursing workload: understanding the variability. Nursing economics, 1997, 15:171-82.

21. Van Gerven R, Delloz H, Sermeus W. Systematic triage in the emergency department using the Australian National triage Scale: a pilot project. European journal of emergency medicine, 2001, 8:3-7.

22. Yousif K, Bebbington J, Foley B. Impact on patients triage distribution utilizing the Australasian Triage Scale compared with its predecessor the National Triage Scale. Emergency medicine Australasia, 2005, 17:429-33.

23. Dann E, Jackson R, Mackway-Jones K. Appropriate categorization of mild pain at triage: a diagnostic study. Emergency nurse, 2005, 13:28-32.

24. Dong SL et al. The effect of training on nurse agreement using an electronic triage system. Canadian journal of emergency medicine, 2007, 9:260-6.

25. Pomerantz, J. Factors Influencing digital reference triage: a think-aloud study. The library quarterly, 2004, 74:235-64.

26. Cooke M, Jinks S. Does the Manchester Triage System detect the critically ill? Journal of accident and emergency medicine, 1999, 16:179-81.

27. Nocera A, Garner A. An australian mass casualty incident triage system for the future based upon triage mistakes of the past: the Homebush Triage Standard. Australian New Zealand journal of surgery, 1999, 69:603-8.

28. Cavallini M et al. Fire disasters in the twentieth century. Annals of burns and fire disasters, 2007, 20(2).

29. Sinclair $L$ et al. How effective are mental health nurses in A\&E departments? Emergency medicine journal, 2006, 23:687-92.

30. Broadbent M, Moxham L, Dwyer T. The development and use of mental health triage scales in Australia. International journal of mental health nursing, 2007, 16:413-21.

31. Wuerz R et al. outcomes are predicted by a new five-level triage algorithm. Academic emergency medicine, 1999, 6:389.

32. Beveridge $\mathrm{R}$ et al. Reliability of the Canadian Emergency Department Triage and Acuity Scale: interrater agreement. Annals ofemergency medicine, 1999, 34:155-9.

33. Wuerz R, Fernandes C, Alarcon J. Inconsistency of emergency department triage. Annals of emergency medicine, 1998, $32: 431-5$
34. Gerdtz M, Bucknall T. Australian triage nurses' decision making and scope of practice. Australasian journal of advanced nursing, 2000, 18:24-33.

35. McMillan JR, Younger MS, DeWine LC. Satisfaction with hospital emergency department as a function of patient triage. Health care management review, 1986, 11:21-7.

36. Salk E et al. Effect of visual cues, vital signs and protocols on triage: a prospective randomized crossover trial. Annals of emergency medicine, 1998, 32:655-64.

37. Richardson D. No relationship between emergency department activity and triage. Academic emergency medicine, 1998, 5:141-5.

38. Andersson A-K, Omberg M, Svedlund M. Triage in the emergency department: a qualitative study of the factors which nurses consider when making decisions. Nursing critical care, 2006, 11:136-45.

39. Wong EML et al. The effect of a triage pain management protocol for minor musculoskeletal injury patients in a Hong Kong emergency department. Australasian emergency nursing journal, 2007, 10:64-72.

40. Chung JYM. An exploration of accident and emergency nurse experiences of triage decision making in Hong Kong. Accident and emergency nursing, 2005, 13:206-13.

41. Fernandez C, Wuerz R, Clark $\mathrm{S}$ et al. How reliable is emergency department triage? Annals of emergency medicine, 1999, 34:141-59.

42. Considine J, Ung L, Thomas S. Triage nurses' decisions using the National Triage Scale for Australian emergency departments. Accident and emergency nursing, 2000, 8:201-9.

43. Wuerz $\mathrm{R}$ et al. Pilot phase reliability of a new five-level triage algorithm. Academic emergency medicine, 1999, 6:398-9.

44. Wuerz $\mathrm{R}$ et al. Reliability and validity of a new five-level triage instrument. Academic emergency medicine, 2000, 7:236-42.

45. Travers DA et al. Five-level triage system more effective than three-level in tertiary emergency department. Journal of emergency nursing, 2002, 28:395-400.

46. Elder $\mathrm{R}$ et al. Patient satisfaction with triage nursing in a rural hospital emergency department. Journal of nursing quality, 2004, 19:263-8.

47. Raper J, Davis BA, Scott L. Patient satisfaction with emergency department triage nursing care: a multicenter study. Journal of nursing care and quality, 1999, 13:11-24.

48. O'Connell JM, Stanley JL, Malakar CL. Satisfaction and patient outcomes of a telephone-based nurse triage services. Managed care, 2001, 10:55-60.

49. Cameron P, Kennedy M, McNeil J. The effects of bonus payments on emergency service performance in Victoria. Medical journal of Australasia, 1999, 171:243-6

50. Kelly A, Richardson D. Training for the role of triage in Australasia. Emergency medicine, 2001, 13:230-2.

51. Ehrenberg G, Ehufors M. Triage in emergency departments: national survey. Journal of clinical nursing, 2005, 14:1067-74.

52. Purnell L. A survey of the qualifications, special training, and levels of personell working emergency department triage. Journal of nursing staff development, 1993, 9:223-6.

53. Kerrison SA, Chapman R. What general emergency nurses want to know about mental health patients presenting to their emergency department. Accident and emergency nursing, 2007, 15:48-55.

54. Clarke DE et al. Education to improve the triage of mental health patients in general hospital emergency departments. Accident and emergency nursing, 2006, 14:210-8.

55. Happell B, Summers M, Pinikahana J. Measuring the effectiveness of the national mental health triage scale in an emergency 
department. International journal of mental health nursing, 2003, 12:288-92.

56. Bindman A. Triage in accident and emergency departments. British medical journal, 1995, 311:404.

57. Brillman JC et al. Triage: limitations in predicting need for emergent care and hospital admission. Annals of emergency medicine, 1996, 27:493-500.

58. Goodacre $\mathrm{S}$ et al. Consistency of retrospective triage decisions as a standardized instrument for audit. Journal of accident and emergency medicine, 1999, 16:322-4.

59. Vertesi L. Does the Canadian Emergency Department Triage and Acuity Scale identify non-urgent patients who can be triaged away from the emergency department? Canadian journal of emergency medicine, 2004, 6:337-42.

60. Oktay $\mathrm{C}$ et al. Appropriateness of emergency department visits in a Turkish University Hospital. Croatian medical journal, 2003, 44:585-91.

61. Fateha BEA, Hamza AY. Impact of triage in accident and emergency departments in Bahrain. Eastern Mediterranean heath journal, 2001, 7:790-8.

62. Huang JA et al. Determining factors of patient satisfaction for frequent users of emergency services in a medical center. Journal of the Chinese Medical Association, 2004, 67:403-10.
63. Miles JA, Naumann SE. The English patient: a model of patient perceptions of triage in an urgent care department in England. Management, 2004, 7:1-11

64. Al-Almaie SM. Ability of adult patients to predict absence or presence of fever in an emergency department triage clinic. Journal of family and community medicine, 1999, 6(1):29-34.

65. Richards DA et al. Nurse telephone triage for same day appointments in general practice: multiple interrupted time series trial of effect on workload and cost. British medical journal, 2002, 325:1214-9.

66. Eminovic $\mathrm{N}$ et al. First evaluation of the NHS Direct online clinical enquiry service: a nurse-led web chat triage service for the public. Journal of medical internet research, 2004, 6:e17.

67. Wynaden $\mathrm{D}$ et al. Emergency department mental health triage consultancy service: a qualitative evaluation. Accident and emergency nursing, 2003, 11:158-65.

68. Kevin J. An evaluation of telephone triage in mental health nursing. Australasian e-journal for the advancement of mental health, 2002, 1:2-12.

\section{The World Health Report 2008 - primary Health Care (Now More Than Ever)}

Why a renewal of primary health care (PHC), and why now, more than ever? Globalization is putting the social cohesion of many countries under stress, and health systems are clearly not performing as well as they could. Few would disagree that health systems need to respond better and faster to the challenges of a changing world. PHC can do that.

As nations seek to strengthen their health systems, they are increasingly looking to PHC to provide a clear and comprehensive sense of direction. The World Health Report 2008 analyses how primary health care reforms, that embody the principles of universal access, equity and social justice, are an essential response to the health challenges of a rapidly changing world and the growing expectations of countries and their citizens for health and health care.

The Report identifies four interlocking sets of PHC reforms that aim to: achieve universal access and social protection, so as to improve health equity; re-organize service delivery around people's needs and expectations; secure healthier communities through better public policies; and remodel leadership for health around more effective government and the active participation of key stakeholders.

The current report is available in 6 languages, including Arabic, and can be ordered from WHO Publications or downloaded at http://www.who.int/whr/2008/en/index.html. 